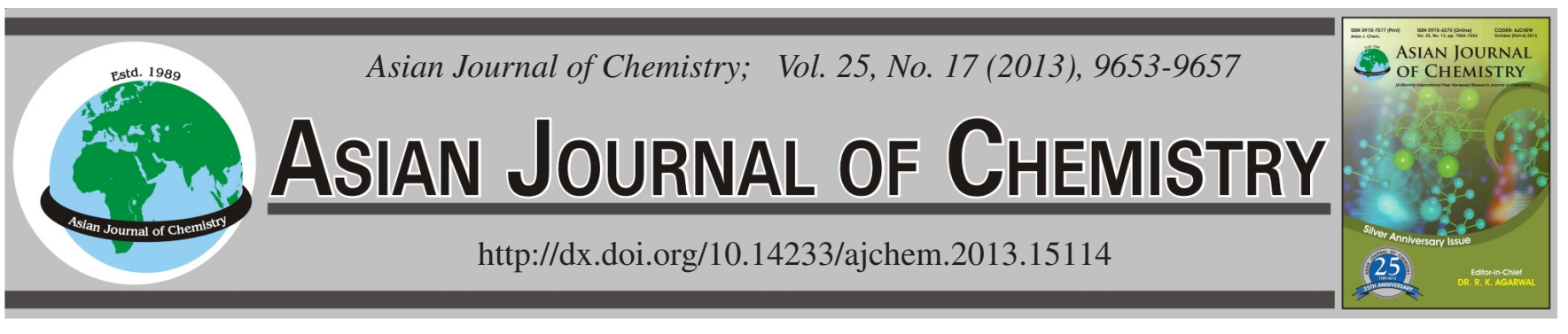

\title{
Optimization and Validation of HPLC-ELSD Method for Determination of Swainsonine in Chinese Locoweeds
}

Jincheng Li, Guodong Yang, Danju Kang, Yanhong Li, Yan Wang, Guoxia Geng and Jianhua Wang*

College of Veterinary Medicine, Northwest A\&F University, Shaanxi, Yangling 712100, P.R. China

*Corresponding author: E-mail: jhwang1948@sina.com

An HPLC method has been optimized and validated for determining swainsonine, a potent glucosidase inhibitor present in locoweeds (any of several plants of the genera Oxytropis and Astragalus in the pea family, which can cause severe poisoning when eaten by livecstock.) of China with isopropanol as a weak eluent. Swainsonine was separated from an extract of locoweeds on hydrophilic interaction chromatography column, using isopropanol- $2 \mathrm{mM}$ ammonium acetate $(52: 48, \mathrm{v} / \mathrm{v})$ as mobile phase at a flow rate of $0.4 \mathrm{~mL} / \mathrm{min}$. During post column detection, swainsonine was detected by evaporative light scattering detector. The method was validated and the detection limit was $6 \mu \mathrm{g} / \mathrm{mL}$. This method is sufficiently sensitive for determining swainsonine in Chinese locoweeds.

Key Words: Swainsonine, Locoweed, Evaporative light scattering detector, Hydrophilic interaction chromatography.

\section{INTRODUCTION}

Swainsonine (SW) (Fig. 1) (1,2,8-trihyroxyoctahydroindolizidine), a plant toxin the main toxic ingredient of major locoweed species (Astragalus and Oxytropis), was isolated initially from the fruit of Australian Swainsona canescens and identified to be an polyhydroxy alkaloid ${ }^{1}$, severely threaten the development of animal husbandry in prairie ${ }^{2,3}$. The swainsonine inhibits lysosomal $\alpha$-mannosidase, Golgi $\alpha$ mannosidase $\mathrm{II}^{4}$ and causes a dual biological effect, a lysosomal storage disorder ${ }^{4,5}$ and interference with the $\mathrm{N}$-linked glycosylation pathway ${ }^{6}$. It's quite specific and reversible inhibition for $\alpha$-mannosidase was used as a tool to investigate the biosynthesis and function of asparagine-linked complex type oligosaccharide moieties of glycoproteins ${ }^{7}$. Since swainsonine was first demonstrated the ability to enhance immune function ${ }^{8}$, there has been constant reports in the past several decades on swainsonine because of its potential use as an anticancer agent and an immunomodulator ${ }^{9-13}$. Swainsonine represents a new class of compounds, natural polyhydroxy alkaloids, that has already demonstrated a wide range of biological activities ${ }^{14}$.
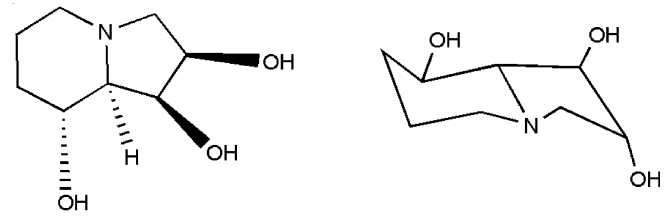

Fig. 1. Chemical structure and chair conformation of swainsonine
As a unique class of $\mathrm{N}$-alkyl substituents alkaloids, it's the special chemical structure that leads to particular chemical properties, spectral properties and remarkable biological properties ${ }^{15}$. Swainsonine can't react with alkaloid-specific chromogenic reagents such as iodoplatinate or Dragendorff's reagent and the ninhydrin colour reaction is insensitive and non-specific ${ }^{16}$. The pyrrole-specific Ehrlich's reagent has been used routinely on thin layer chromatographic plates for the visualization of swainsonine existed in Astragalus and Oxytropis species after the bicyclic indolizidine ring system was dehydrated with acetic anhydride by heating ${ }^{17}$. There exists some difficulty in applying swainsonine by using routine detector such as UV-visible detector because there is no chromophore for swainsonine to take spectrographic analysis ${ }^{14}$. Therefore, to draw on the experience of analyzing sugars, a derivatization procedure was used for GC in order to give efficient absorption and fluorescent properties ${ }^{18}$. The HPLC is seldom used in routine analysis unless in tandem with mass spectrometer because of its high polar and can't be retained in normalphase HPLC or reversed-phase HPLC ${ }^{14}$.

With increasing technological sophistication, GC and HPLC, as important analytical technique, have received frequent usage to analyze the swainsonine. The invention of ELSD, a semi-universal detector that can detect any nonvolatile analyte, makes it possible to analyze compounds with no chromophore sensitively and directly. And the HILIC makes up the deficiency of NP-HPLC and RP-HPLC for retention of high polar compounds. Therefore, in this study, we focused 
on reassessing the retention behaviours of swainsonine and optimizing the separation approach for determining swainsonine present in Chinese locoweeds and other related products and discussed the efficient separation of swainsonine with HILIC.

\section{EXPERIMENTAL}

Swainsonine (Cat. 86204) was purchased from SigmaAldrich (St. Louis, MO) and used as a reference standard in determination experiments. Isopropanol (HPLC grade) was purchased from Tianjin Kermel Chemical Reagent Co., Ltd (Tian Jin, China), ammonium acetate from Tianjin Bodi Chemical Holding Co., Ltd. (Tian Jin, China). Ultra-pure water was produced by Milli-Q system (Millipore, Bedford, MA, USA). All solvents and samples were filtered through 0.22 $\mu \mathrm{m}$ filter (Xinya Co., Shanghai, China) before injecting into the HPLC.

Preparation of samples: Locoweeds were selected from western rangelands in China (Table-1). Finely ground, air-dried plant material was milled to 40 mesh powder as extraction sample and dried at $70-80{ }^{\circ} \mathrm{C}$ for $24 \mathrm{~h}$ before extracted.

Instruments and chromatography: The HPLC system used consist of a Waters 1525 binary HPLC pump, a column temperature controller and a Waters 2424 evaporative light scattering detector (Waters Corp, USA). The separation was performed using a mixture of isopropanol -2 $\mathrm{mM}$ ammonium acetate $(52: 48, \mathrm{vol} / \mathrm{vol})$ as mobile phase by an X-Bridge HILIC column (150 $\mathrm{mm} \times 4.6 \mathrm{~mm}, 3.5 \mu \mathrm{m}$ particle size, Waters Corp, USA). The flow rate was adjusted to $0.4 \mathrm{~mL} / \mathrm{min}$ and the column temperature was maintained at $38{ }^{\circ} \mathrm{C}$. The injection volume was $20 \mu \mathrm{L}$. ELSD conditions were optimized in order to achieve maximum sensitivity; the temperature of the drift tube was set at $50{ }^{\circ} \mathrm{C}$, nitrogen gas was used as the nebulizing gas at a pressure of 25 psi and the gain was usually set at 100 . The peak areas were registered using Waters Breeze 2 software.

Standard calibration curves of swainsonine: The swainsonine standard was weighed accurately and dissolved in methanol, then prepared for a stock solution containing $1 \mathrm{mg} / \mathrm{mL}$ of swainsonine and stored at $-20^{\circ} \mathrm{C}$ until analysis. The standard solutions were prepared by dilution of the stock working solution with the mobile phase to reach the concentration range of $10-100 \mu \mathrm{g} / \mathrm{mL}$ for swainsonine. Triplicate $20 \mu \mathrm{L}$ injections were made for each concentration and chromatographed under the conditions described above and a calibration curve was made.

Extraction and quantification: Swainsonine extraction and quantification were measured using a modification of a previously published procedure ${ }^{17}$ in the following manner. Plant material was weighed accurately and extracted with methanol in a Soxhlet apparatus. The extract was evaporated to dryness. The residue dissolved in $10 \mathrm{~mL}$ of $2 \%$ acetic acid, filtered and applied to a Dowex 50W-X8 ion exchange column.The volume of prewashed ion-exchange resin in glass column is $10 \mathrm{~mL}$. The column was washed with distilled water and then eluted with $1 \mathrm{~mol} / \mathrm{L}$ aqueous ammonium hydroxide. The eluate was evaporated to dryness and redissolved in $1 \mathrm{~mL}$ methanol. The sample solutions were prepared by dilution of the working solution with the mobile phase to reach the concentration range of $10-100 \mu \mathrm{g} / \mathrm{mL}$ for swainsonine before analysis.

Optimization of the chromatographic system: To effectively separate swainsonine from other compounds in the locoweeds sample, various HPLC mobile phase systems were tested. All the analysis shows that the optimal mobile phase was isopropanol-2 $\mathrm{mM}$ ammonium acetate (52:48, v/v) which was employed in the following experiments (Fig. 2a). Identification of swainsonine was based on retention time, co-injection with standard (Fig. 2b).

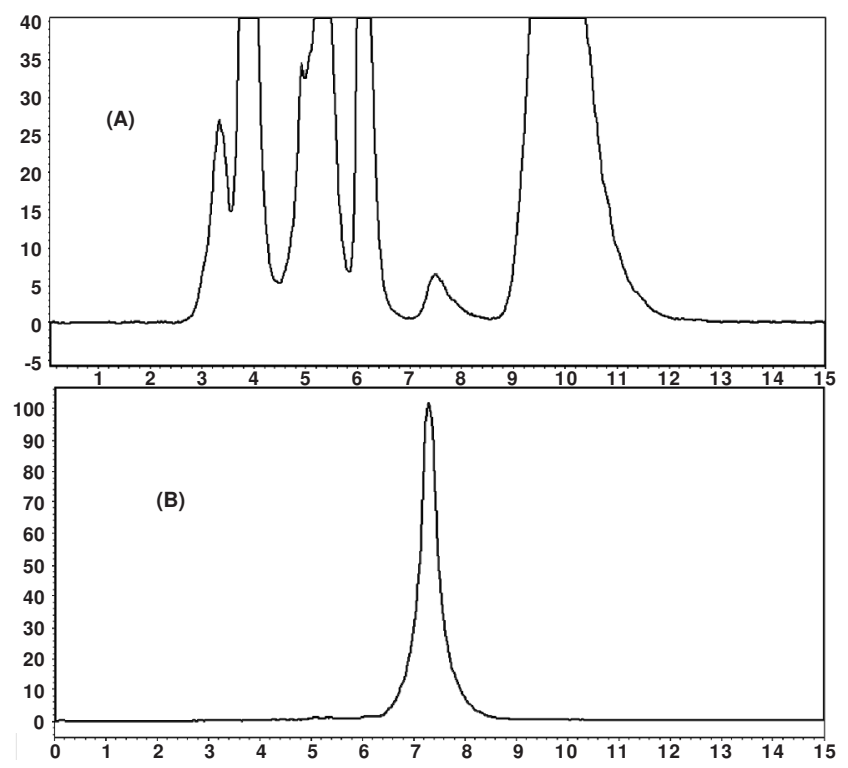

Fig. 2. Representative HPLC-ELSD chromatogram of swainsonine from locoweeds (A) and chromatogram of standard swainsonine (B)

Method validation: The method was validated in agreement with the International Conference on Harmonization Guidelines (ICH, 1996) with regards to its specificity, linearity, limits of detection (LOD), limits of quantification (LOQ), accuracy and precision.

\section{RESULTS AND DISCUSSION}

\section{Optimization of the chromatographic system}

Effect of the column type: We first attempted to seek the most appropriate column that would enable swainsonine, which is a highly polar compound to be retained. Ligand

\begin{tabular}{|c|c|c|c|c|}
\hline \multicolumn{5}{|c|}{$\begin{array}{c}\text { TABLE-1 } \\
\text { SELECTED LOCOWEED SPECIES IN CHINA }\end{array}$} \\
\hline Locoweed species & Location & GPS & Collection date & Vegetation period \\
\hline O. glabra & Pingluo, Ningxi & N 3849'27"; W 106³8'24" & September 2008 & Podding \\
\hline O. sericopetala & Qushui, Tibet & N 29¹9'16"; W 9046'33" & August 2005 & Podding \\
\hline O. ochrocepala & Haiyu an, Ningxi & N 36³0'01"; W $105^{\circ} 37^{\prime} 24^{\prime \prime}$ & August 2007 & Podding \\
\hline O. kansuensis & Tianzhu, Gans & N 36 $58^{\prime} 29^{\prime \prime} ;$ W $102^{\circ} 58^{\prime} 28^{\prime \prime}$ & September 2006 & Podding \\
\hline O. glacialis & Ali, Tibet & N 32॰27'14"; W 80²1"43" & August 2005 & Podding \\
\hline
\end{tabular}


exchange and aminopropyl columns are commonly applied for HPLC determination of relatively polar compounds such as carbohydrates and water soluble vitamins. However, several reversed-phase columns were tested under various conditions but reasonable retention was not obtained for standard swainsonine. Finally, assays were tried using the HILIC column, which was designed as an efficient tool to retain and separate highly polar compounds and its analytical application for carbohydrates and peptide has been reported. Hence, it seemed logical to anticipate that a hydrophilic interaction liquid chromatography column might be a desirable column for efficient separation of swainsonine.

Effect of the strength and composition of mobile phase: Mobile phase strength and composition are important parameters in HILIC separation. The effect of acetone, acetonitrile, methanol or isopropanol was examined as a weak eluent and water or aqueous buffer as a strong eluent although acetonitrile is used as a traditional HILIC mobile phase on account of its low strength, low viscosity, excellent dissolving capacity and low UV absorbance. The typical retention behaviours and separation were not observed on acetone or methanol because of low resolution (Fig. 3a,c). The retention times were observed to be inversely proportional to the water content in the eluent but hardly improve the resolution effectively. Acetonitrile and isopropanol were selected for further investigation.

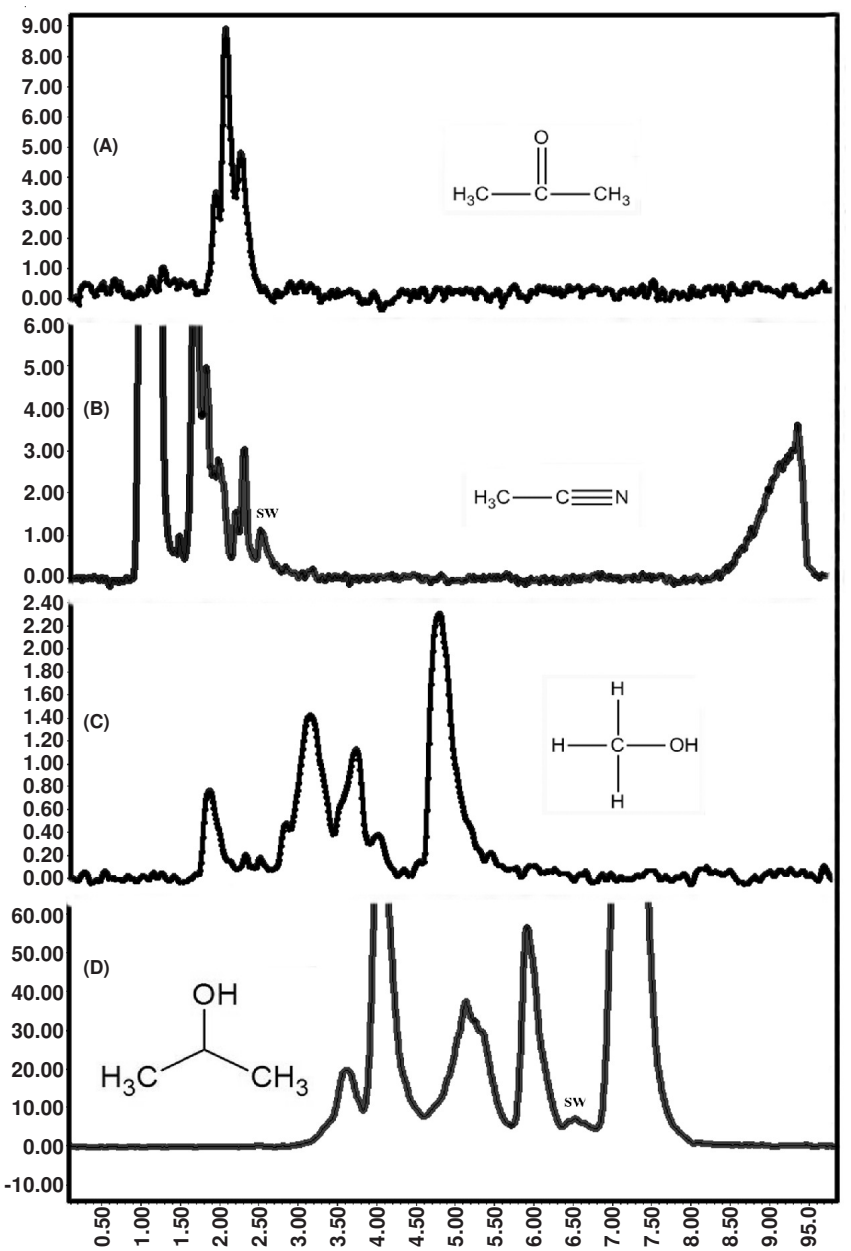

Fig. 3. Effect of the strength and composition of mobile phase: swainsonine was separated from the other peak components when use acetonitrile (B) and isopropanol (D). No single distinct peak of swainsonine was observed in acetone $(\mathrm{A})$ or methanol $(\mathrm{C})$
Effect of ionic strength and pH of mobile phase: Ionic strength can affect the retention and selectivity of polar compounds in HILIC. For extremely polar analytes, high concentrations of buffer are required to assure that the analyte will be in a single ionic form. In consideration of specific requirements on ELSD, ammonium acetate was used to reform ionic strength of mobile phase in this investigation. Changing the concentration of ammonium acetate solution can significantly effect on the resolution of swainsonine (Fig. 4). As the concentration increased between 1 and $10 \mathrm{mmol} / \mathrm{L}$, the retention time of swainsonine was prolonged and a visible change was occurred on the resolution. In all separations, the elution order was the same, but the best peak shape and resolution appeared at $2 \mathrm{mM}$ ammonium acetate solution mixed with isopropanol. The $\mathrm{pH}$ of mobile phase is an important parameter to be optimized as it affects the ionization of compounds. Acetic acid and aqueous ammonia were evaluated as additives and seldom effect was observed but erratic baseline. The swainsonine was not achieved baseline separation under the optimized condition of acetonitrile (Fig. 3b).

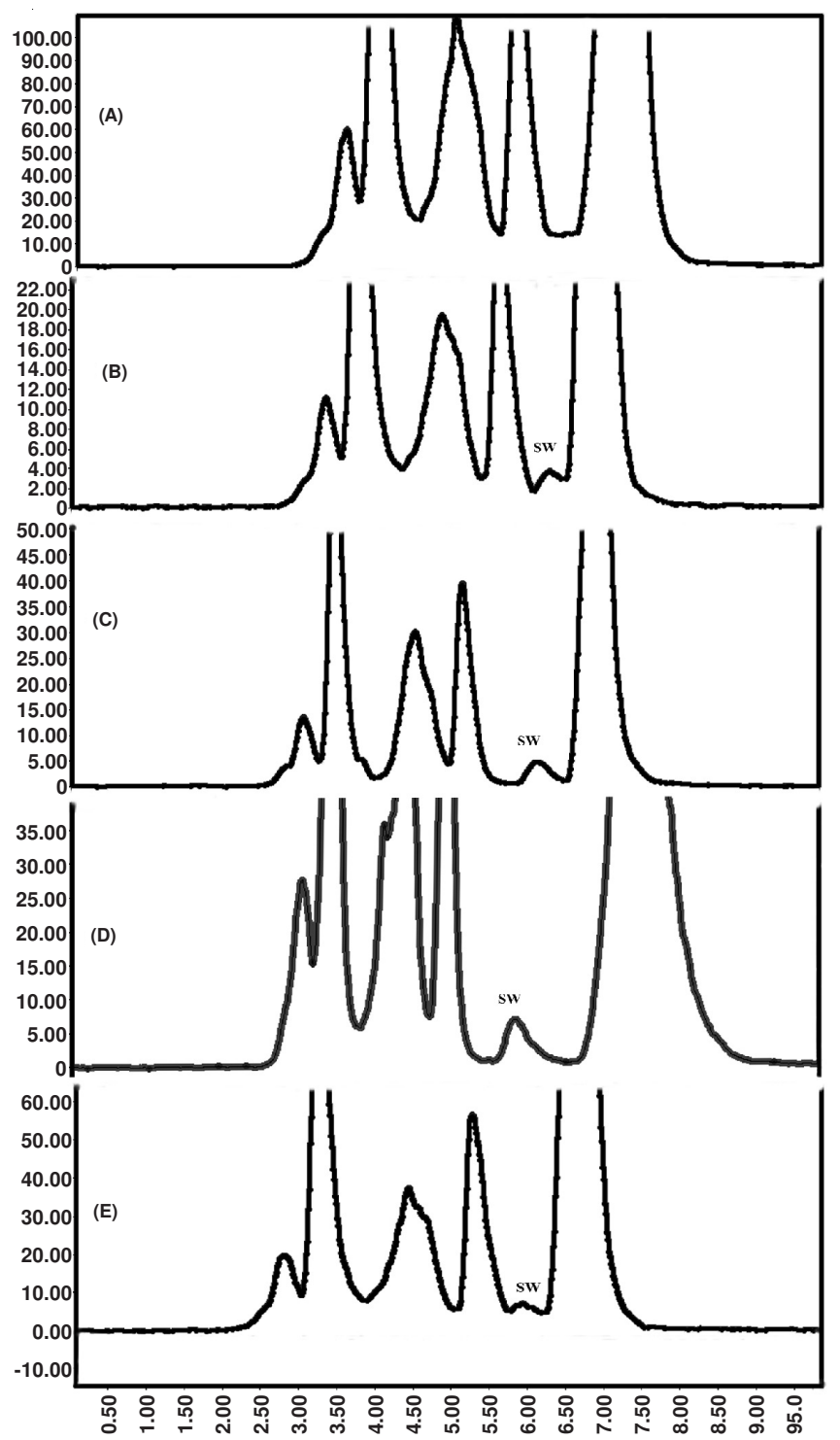

Fig. 4. Effect of ionic strength: the concentration of ammonium acetate solution (A) $10 \mathrm{mmol} \mathrm{L} \mathrm{(B)} 5 \mathrm{mmol} \mathrm{L} \mathrm{(C)} 3.5 \mathrm{mmol} \mathrm{L}$ (D) $2 \mathrm{mmol}$ $\mathrm{L}$ (E) $1 \mathrm{mmol} \mathrm{L}$ 
Effect of the flow rate and column temperature: Flow rate of mobile phase has important effect on retention time, peak area and little effect on separation. Column temperature is an important parameter that affects the retention and separation of analytes in HILIC. These two factors were assessed within the allowable range of column pressure based on the high viscosity of isopropanol. Comprehensive consideration, the flow rate was adjusted to $0.4 \mathrm{~mL} / \mathrm{min}$ and the column temperature was maintained at $38^{\circ} \mathrm{C}$.

Optimization of detection conditions: ELSD conditions were optimized in order to achieve maximum sensitivity. The nebulizer-gas flow rate and drift temperature are the main instrumental parameters affecting signal response. The drift temperature was tested at $40-65^{\circ} \mathrm{C}$ to study the influence on ELSD response and signal-to-noise ratio. The best result was obtained with a drift tube temperature at $50{ }^{\circ} \mathrm{C}$ and nebulizing gas pressure at 25 psi.

Mass spectra validation: ELSD enabled the detection of standard swainsonine as a single distinct peak with a retention time of $7.3 \mathrm{~min}$. Locoweeds swainsonine was eluted as a defined peak at a retention time of $7.3 \mathrm{~min}$ with the baseline separated from the other peak components (Fig. 2a). There is no tailing for the locoweeds swainsonine peak (Fig. 2b) and its purity was checked by HPLC/MS. This peak (7.3 min) was identified as swainsonine on the basis of the MS profile (Fig. 5b,c). When the prepared sample was subjected to HPLC coupled to MS using an X-Bridge HILIC column, a well-defined peak ascribed to swainsonine was detected at a suitable retention time (7.19 min) in the total ion current chromatogram. This peak component gave a molecular ion $[\mathrm{M}+\mathrm{H}]^{+}$at $m / z 174.2 \mathrm{amu}$, which corresponds to swainsonine (Fig. 5c). The accumulated spectrum contained only one swainsonine peak $\left([\mathrm{M}+\mathrm{H}]^{+}\right.$at $\mathrm{m} / \mathrm{z} 174.2 \mathrm{amu}$ ) and no apparent peaks for the impurities. This indicated that no apparent peaks for the impurities with the locoweeds swainsonine peak.

Method validation: The method was validated in terms of linearity, precision, limits of detection (LOD) and quantification (LOQ), stability, repeatability and the recovery test.

Linearity: The calibration curves were constructed by plotting the peak areas versus the concentration $(\mu \mathrm{g} / \mathrm{mL})$ of each standard solution after log-transformation. The data provided a linear calibration following the equation: $\mathrm{Y}=\mathrm{A}+\mathrm{B}$ $\mathrm{X}$, where $\mathrm{Y}$ is the logarithm (base e) of the peak area, $\mathrm{X}$ is the logarithm (base e) of sample concentration, $\mathrm{A}$ is the intercept and $\mathrm{B}$ is the slope. The regression equation was $\mathrm{Y}=7.8998+$ $1.0617 \mathrm{X}$ and the regression coefficient $\mathrm{r}^{2}$ was 0.9974 .

Precision and accuracy: The precision injection was evaluated by repeated injection of the sample solution six times.
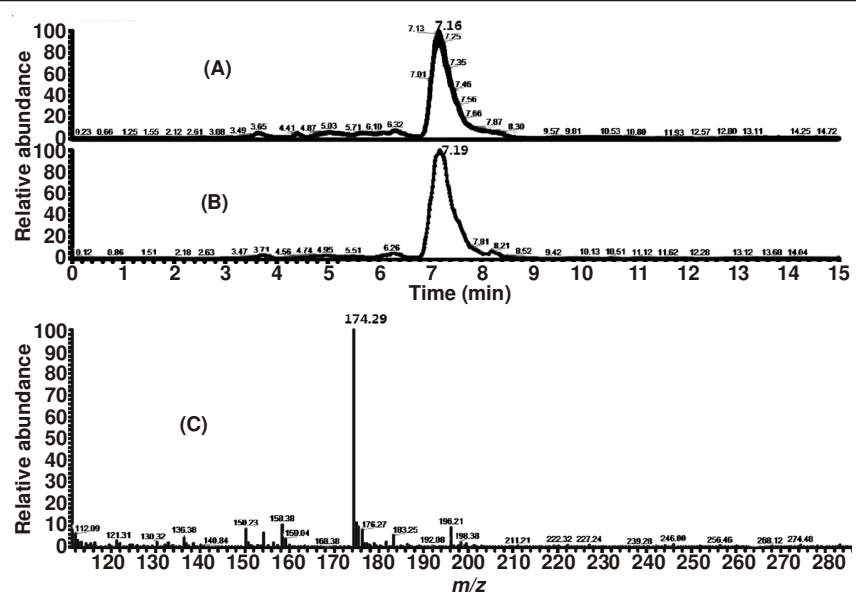

Fig. 5. HPLC-MS analysis of swainsonine (SW) present in locoweeds: (A) Total ion chromatograpy (TIC); (B) Extract ion chromatograpy (EIC) to the $[\mathrm{M}+\mathrm{H}]^{+}$ion of swainsonine $(\mathrm{mz} \mathrm{174.2)}$; (C) MS spectrum of the peak detected between 7.0 and 8.0

The relative standard deviation value (RSD) of peak area and retention time are 3.56 and $0.32 \%(n=6)$, respectively. Sample intraday and interday stability were monitored by analysing the same sample solution within 1 day at $0,4,8 \mathrm{~h}$ and for 3 days at an interval of every $24 \mathrm{~h}$, respectively. The RSD values of intraday stability were $0.26-2.51 \%$ and those of interday stability were $0.28-1.88 \%$ (Table-2).

\section{TABLE-2}

INTRA- AND INTER-DAY PRECISION AND ACCURACY

\begin{tabular}{cccc}
\hline $\begin{array}{c}\text { Actual } \\
\text { concentration } \\
(\mu \mathrm{g} / \mathrm{mL})\end{array}$ & $\begin{array}{c}\text { Detected } \\
\text { concentration } \\
\left(\text { mean } \pm \text { S.D. }{ }^{\mathrm{a}}, \mathrm{n}=3\right)\end{array}$ & $\begin{array}{c}\text { R.S.D. } \\
(\%)\end{array}$ & $\begin{array}{c}\text { Accuracy } \\
\left(\mathrm{RD}^{\mathrm{c}}\right)(\%)\end{array}$ \\
\hline \multicolumn{4}{c}{ Intra-day } \\
\hline 20 & $20.32 \pm 0.05$ & 0.26 & 1.88 \\
80 & $76.10 \pm 1.96$ & 2.51 & 2.03 \\
100 & $104.86 \pm 0.94$ & 0.90 & 3.94 \\
\hline \multicolumn{4}{c}{ Inter-day } \\
\hline 80 & $20.35 \pm 0.05$ & 0.28 & 1.56 \\
100 & $73.16 \pm 0.94$ & 1.28 & 7.42 \\
\hline
\end{tabular}

A recovery test was used to evaluate the accuracy of the method by using an external standard method. The known amount of swainsonine was added into the samples to ascertain the recovery of swainsonine from locoweeds and the resultant solutions were analyzed by the valid HPLC method. The recovery of swainsonine was between 96.06 and $108.58 \%$ and RSD was between 1.02 and $6.70 \%$ (Table-3).

Limit of quantification (LOQ) and limit of detection (LOD): The LOD and LOQ under the present chromatographic

\begin{tabular}{|c|c|c|c|c|c|c|c|c|c|c|c|}
\hline \multicolumn{12}{|c|}{$\begin{array}{c}\text { TABLE-3 } \\
\text { RECOVERY STUDIES OF SWAINSONINE ADDED TO SELECTED SAMPLES OF LOCOWEEDS }\end{array}$} \\
\hline Analytes & $\begin{array}{c}\text { Contents }(\mu \mathrm{g}) \\
(\text { mean } \pm \text { S.D. } \\
n=3)\end{array}$ & $\begin{array}{l}\text { Added } \\
\text { amount } \\
(\mu \mathrm{g})\end{array}$ & $\begin{array}{l}\text { Recorded } \\
\text { amount } \\
(\mu \mathrm{g})\end{array}$ & \multicolumn{3}{|c|}{ Found $(\mu \mathrm{g})$} & \multicolumn{3}{|c|}{ Recovery (\%) } & $\begin{array}{c}\text { Average } \\
\text { recovery } \\
(\%)\end{array}$ & $\begin{array}{r}\mathrm{RSD}^{\mathrm{b}} \\
(\%)\end{array}$ \\
\hline \multirow{3}{*}{ O. glacialis } & \multirow{3}{*}{$9.48 \pm 0.48$} & 5 & 14.48 & 14.21 & 14.25 & 14.38 & 94.64 & 95.42 & 98.11 & 96.06 & 1.89 \\
\hline & & 10 & 19.48 & 19.42 & 19.35 & 19.22 & 99.40 & 98.69 & 97.40 & 98.50 & 1.02 \\
\hline & & 15 & 24.48 & 26.07 & 23.98 & 25.08 & 110.63 & 96.72 & 104.04 & 103.79 & 6.70 \\
\hline \multirow{3}{*}{ O. sericopetala } & \multirow{3}{*}{$18.96 \pm 0.96$} & 10 & 28.96 & 29.72 & 29.10 & 30.06 & 107.66 & 101.45 & 111.06 & 106.72 & 4.56 \\
\hline & & 20 & 36.96 & 39.82 & 41.64 & 40.56 & 104.32 & 113.42 & 108.01 & 108.58 & 4.21 \\
\hline & & 30 & 48.96 & 51.18 & 49.42 & 49.44 & 107.40 & 101.55 & 101.62 & 103.53 & 3.24 \\
\hline
\end{tabular}


conditions were determined at signal-to-noise ratios $(\mathrm{S} / \mathrm{N})$ of 3 and 10, respectively. The measured LOQ and LOD values of the described method were observed for 20 and $6 \mu \mathrm{g} / \mathrm{mL}$, respectively, which will meet the requirements of validation for a new assay protocol.

Application: To apply the present method for determining locoweed swainsonine, its standard curve was made. The standard curve of swainsonine using ELSD was not linear, which is a common feature of this detector ${ }^{19}$. The concentration of solution is well correlated with chromatographic peak area; and a mean correlation coefficient $r^{2}=0.997$. The detection limit was $6 \mu \mathrm{g} / \mathrm{mL}$ for swainsonine at a signal-to-noise ratio of 3. Hence, we can see that the sensitivity of the present ELSD method for swainsonine is relatively high. By using this standard curve, the concentrations of swainsonine in three cultivars of locoweeds were defined as $0.932-1.580 \mathrm{mg} / 100 \mathrm{~g}$ (0.0009-0.0015\%). Without recourse to cultivars, the present method showed enough sensitivity to determine swainsonine concentration.

\section{Conclusion}

As a typical naturally occurring polyhydroxy alkaloids and azasugars, swainsonine is carbohydrate analogs in which the ring oxygen has been replaced with nitrogen and found to be widespread in plants and microorganisms. These structural features render the compounds highly polar and highly water soluble. To determine swainsonine by HPLC, it can't be retained in normal-phase HPLC or reversed-phase HPLC. Hydrophilic interaction liquid chromatography (HILIC) mode has been studied extensively as an analytical tool for the analysis of polar compounds in the past few years. Acetonitrile was used as a weak eluent in a typical mobile phase for HILIC chromatography and water or aqueous buffer as a strong eluent. It is commonly believed that the mobile phase forms a waterrich layer on the surface of the polar stationary phase against the water-deficient mobile phase, creating a liquid-liquid extraction system in HILIC mode ${ }^{20}$. However, polar analytes achieve retention and separation primarily by many pattern interact on each other. Hydrogen bonding probably also contributes to the retention mechanism in HILIC as well as weak electrostatic mechanisms under the high organic solvent conditions used for retention. Mobile phase with its strong ability for hydrogen bonding might disturb the formation of the water layer on HILIC such as acetone and methanol (Fig. 3a,c). The presence of buffering salts in the mobile phase can decrease electrostatic interactions through disruption (Fig. 4). Thus, HILIC is more than just simple partitioning between a waterenriched layer on the surface of a polar stationary phase and a mobile phase that has a high percentage of organic solvent. Because the retention mechanism of HILIC mode using isopropanol as a weak eluent was poorly understood, we performed the systematic evaluation of the separation parameters during the method development, which included mobile phase strength, composition, ionic strength, $\mathrm{pH}$, flow rate and column temperature. The results showed that the retention and resolution of swainsonine was achieved mainly by hydrogen-bond and electrostatic mechanisms.

As mentioned above, the HPLC-ELSD method was reassessed and optimized. Chromatographic separation was performed from an extract of locoweeds by using isopropanol as a weak eluent. The results from this work clearly show the optimization and validation of a new, rapid and simple quantitative determination method for swainsonine present in locoweeds. In contrast, our HPLC-ELSD method enables the simple and convenient determination of swainsonine without any labeling procedures. This is a great advantage when multiple samples are analyzed.

\section{ACKNOWLEDGEMENTS}

The authors acknowledged the National Natural Science Foundation of China, project number 30871901, for financial support.

\section{REFERENCES}

1. S.M. Colegate, P.R. Dorling and C.R. Huxtable, Aust. J. Chem., 32, 2257 (1979).

2. R.J. Molyneux and L.F. James, Science, 216, 190 (1982).

3. K.E. Panter, L.F. James, B.L. Stegelmeier, M.H. Ralphs and J.A. Pfister, J. Nat. Toxins, 8, 53 (1999).

4. P.R. Dorling, C.R. Huxtable and S.M. Colegate, Biochem. J., 191, 649 (1980).

5. C.R. Huxtable and P.R. Dorling, Acta Neuropathol., 68, 65 (1985).

6. U. Fuhrmann, E. Bause, G. Legler and H. Ploegh, Nature, 307, 755 (1984).

7. K. Olden, P. Breton, K. Grzegorzewski, Y. Yasuda, B.L. Gause, O.A. Oredipe, S.A. Newton and S.L. White, Pharmacol. Ther., 50, 285 (1991).

8. T. Kino, N. Inamura, K. Nakahara, S. Kiyoto, T. Goto, H. Terano, M. Kohsaka, H. Aoki and H. Imanaka, J. Antibiot. (Tokyo), 38, 936 (1985).

9. L. E. Fellows, G. C. Kite, R. J. Nash, M. Simmonds and A. M. Scofield, Recent Adv. Phytochem., 23, 395 (1989).

10. R. Pili, J. Chang, R.A. Partis, R.A. Mueller, F.J. Chrest and A. Passaniti, Cancer Res., 55, 2920 (1995).

11. A.A. Watson, G.W.J. Fleet, N. Asano, R.J. Molyneux and R.J. Nash, Phytochemistry, 56, 265 (2001).

12. N. Asano, Glycobiology, 13, 93R (2003).

13. R.J. Nash, A. Kato, C.Y. Yu and G.W. Fleet, Future Med. Chem., 3, 1513 (2011).

14. R.J. Molyneux, D.R. Gardner, L.F. James and S.M. Colegate, J. Chromatogr. A, 967, 57 (2002).

15. N. Araújo, S.F. Jenkinson, R.F. Martínez, A.F.G. Glawar, M.R. Wormald, T.D. Butters, S. Nakagawa, I. Adachi, A. Kato, A. Yoshihara, K. Akimitsu, K. Izumori and G.W.J. Fleet, Org. Lett., 14, 4174 (2012).

16. L.D. Hohenschutz, E.A. Bell, P.J. Jewess, D.P. Leworthy, R.J. Pryce, E. Arnold and J. Clardy, Phytochemistry, 20, 811 (1981).

17. R.J. Molyneux, L.F. James, K.E. Panter and M.H. Ralphs, Phytochem. Anal., 2, 125 (1991).

18. W.E. Allen, in Gas chromatographic analysis of the indolizidine alkaloid swainsonine in solutions of ovine origin, New Mexico State University Press, New Mexico, United States, pp. 320 (2006).

19. K. Petritis, C. Elfakir and M. Dreux, J. Chromatogr. A, 961, 9 (2002).

20. B. Buszewski and S. Noga, Anal. Bioanal. Chem., 402, 231 (2012). 